\title{
Rubella Cases Detection through Measles Surveillance System from 2012 to 2016 in Côte d'Ivoire
}

\author{
Christophe Kouakou Nguessan ${ }^{1 *}$, Serge Kouadio Agbo², Youssouf Traore ${ }^{3}$, Cyrille Gourmanon ${ }^{2}$, Dan- \\ iel Kouadio Ekra ${ }^{1}$ and Simplice Ncho Dagnan ${ }^{3}$
}

${ }^{1}$ National Expand Program on Immunization, Cote d'Ivoire

${ }^{2}$ University of Abidjan, Cote d'Ivoire

${ }^{3}$ National Institute Public Hygiene, Cote d'Ivoire

*Corresponding author: Christophe Kouakou Nguessan, National Expand Program on Immunization, Cote d'Ivoire, Tel: +225-0256-2000, E-mail: drchristophe.nguessan@gmail.com

\begin{abstract}
Background: Rubella is a contagious disease caused by a virus. Rubella is a major cause of miscarriage or serious birth defects in a developing baby if a woman is infected while she is pregnant. Côte d'Ivoire is a country that is not immunizing against rubella in its Expend program on immunization (EPI).

In Côte d'Ivoire, suspected cases tested negative and undetermined in the measles case-based surveillance system, are screened for rubella infection.

Our study aims to describe the rubella epidemiological situation through measles surveillance and associated risk factors in Côte d'Ivoire.

Method: An analytical, cross-sectional study was conducted using the Côte d'Ivoire EPI data from 2012 to 2016 . Univariate analysis was performed to summarize characteristics of Rubella suspected and confirmed cases. Bivariate and multivariable analysis were performed to compare rubella cases to non-Rubella cases.

Result: In Côte d'Ivoire, from 2012 to 2016, the National Expanded Program on Immunization (EPI) were notified 3693 Rubella suspected cases in which 615 were confirmed cases. The median age of confirm cases was 6.8 years and the interquartile range was 4.2 to 10 years. The majority of cases were males $(53.8 \%)$.

In multivariate analysis conducted, we found that children aged of 5 to 14 years had high risk of rubella compared to children under 5 [OR 3.31, 95\% Cl $(2.73-4.00)]$. The difference between subjects aged 15 and above and children under 5 -years-old were not significant, [OR of $1.32,95 \% \mathrm{Cl}(0.92$ 1.88)]. In this model, people living in urban areas had a higher risk compared to people living in rural areas [OR $1.22,95 \% \mathrm{Cl}$ $(1.01-1.46)]$ and this difference was significant.
\end{abstract}

Of the 284 Rubella confirmed female cases, $8.5 \%$ were of reproductive age. Children of 5-14 years-old, adults and people living in urban areas were at high risk of rubella.

Conclusion: As the surveillance of rubella Cote d'Ivoire is not using the WHO recommended Rubella case definition, this system could miss some rubella cases. However, our study revealed the burden and trends of rubella in Côte d'Ivoire. Rubella surveillance could be suboptimal in Côte d'Ivoire as cases are under-reported. CRS is not under surveillance. Our data suggests that the country should establish a surveillance system for rubella and CRS, and introduce the rubella vaccine in the national EPI.

\section{Introduction}

Rubella is a contagious viral infection caused by Rubella virus, a Togavirus, genus Rubivirus. In countries without implementation of vaccination control program, Rubella infection is major cause of miscarriage and birth defect [1]. Rubella infection is usually benign that most often affects children and young adults. In rare cases, it can cause serious problems, including brain infections and bleeding. The most serious complication of rubella infection is the damage that it can cause to the developing fetus during pregnancy. If an unvaccinated pregnant woman is infected with rubella virus, she may have a miscarriage or her baby may die just after birth. Also, she may transmit the virus to her developing baby who may develop serious congenital anomalies such as heart problems, hearing and vision loss, intellectual impairment and liver or spleen damage [2].

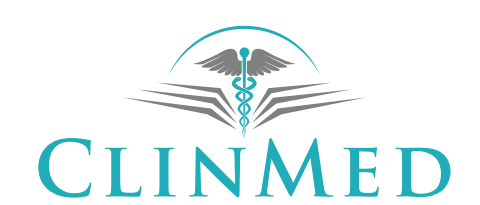

INTERNATIONAL LIBRARY
Citation: Nguessan CK, Agbo SK, Traore Y, Gourmanon C, Ekra DK, et al. (2018) Rubella Cases Detection through Measles Surveillance System from 2012 to 2016 in Côte d'Ivoire. Int J Trop Dis 1:001. Received: March 26, 2018; Accepted: May 14, 2018; Published: May 16, 2018

Copyright: (C) 2018 Nguessan CK, et al. This is an open-access article distributed under the terms of the Creative Commons Attribution License, which permits unrestricted use, distribution, and reproduction in any medium, provided the original author and source are credited. 
Severe congenital anomalies are more common when a woman is infected at the beginning of her pregnancy, especially during the first trimester. These severe malformations are known as congenital rubella syndrome (CRS) [2]. World Health Organization (WHO) estimates that 100,000 children per year are born with CRS [1]. In 2014, 161 WHO member states notified 33,068 cases of rubella against 102 countries for 670,894 in 2010 [3].

For millions of mothers and their children in developing countries, rubella is an ongoing threat. Africa and Southeast Asia have the highest number of estimated CRS cases and the lowest uptake of rubella-containing vaccine. The human and economic toll of rubella in those regions are high [4].

For the while, rubella related CRS is not under surveillance in the Côte d'Ivoire National Expanded Program on Immunization (EPI) system. In Côte d'Ivoire, the National EPI does not include vaccination against rubella in its vaccination schedule (routine and campaign). The last campaigns (2011 and 2014) against measles were organized with the vaccine against measles alone.

In the measles case-based surveillance, suspect measles cases tested negative and undetermined for measles are screened for rubella infection in Côte d'Ivoire. Furthermore, for several years, measles epidemiological surveillance data, showed a large number of rubella cases compared to the measles which is in net decline.

Our study aims to describe the epidemiology of rubella infection through measles case based surveillance and associated risk factors in Côte d'Ivoire.

\section{Methods}

\section{Study design}

This study was an analytical, cross-sectional, based on epidemiological surveillance data of measles and rubella in Côte d'Ivoire conducted by the National EPI from 2012 to 2016.

\section{Study framework, study population and sampling strategy}

Our study concerned all the health districts (82) in Côte d'Ivoire that notified and sent specimen of suspect measles cases to national level (National EPI and Pasteur Institute of Côte d'Ivoire (IPCI), a WHO accredited laboratory for measles surveillance) as part of routine measles case-based surveillance.

The study population was all suspects' cases of rubella in Côte d'Ivoire which were distributed in the 82 health districts of country.

Suspected cases of measles are defined as any person with fever, a generalized maculopapular rash (non-vesicular) and one these signs (cough or cold or conjunctivitis), or anyone in whom a doctor suspects measles [5].

Suspected case of rubella is a suspected case of mea- sles tested negative or indeterminate for the detection of IgM antibodies against measles and then tested for IgM against rubella.

Confirmed case of rubella is a suspected case of rubella whose test shows the presence of IgM antibodies against rubella.

Anyone on the national territory during the study period from January 2012 to December 2016 and who is a suspect of measles but negative or indeterminate case for the search IgM antibodies against measles is likely to be part of our study population.

\section{Sample size}

All suspected and confirmed cases of rubella notified during the 5 years of our study are part of our study. From 2012 to 2016, 3693 suspected cases of rubella were notified.

\section{Criteria of inclusion and exclusion}

To be eligible, study participants must meet the following criteria:

Inclusion criteria: Suspected cases of rubella:

- Be notified as a suspect case of measles.

- Negative or indeterminate for measles IgM antibodies in serum.

- To be tested as a suspected case of rubella.

\section{Confirmed cases of rubella}

- Be positive for IgM antibodies against rubella.

Exclusion criteria: Any subject of the Cote d'Ivoire population who does not meet the criteria above is not part of our study sample.

\section{Procedures}

\section{Data collection}

Data from our study were collected from January 2012 to December 2016 in all 82 health districts of Côte d'Ivoire during case-by-case surveillance of measles by health workers and epidemiological surveillance officers from the generic form of diseases notification (surveillance). This data is collected during an interview with the patient or their parents/care givers. Also for each patient, a blood sample was taken as part of the case-based surveillance of measles in the framework of Integrated Disease Surveillance and Response (IDSR). Samples and notification forms are forwarded to DCPEV, which transfers them to IPCI laboratory for the completion of the biological examinations. The data on the notification forms and the laboratory results are entered in Epi Info software at DCPEV.

Key variables are: Age, sex, home (urban/rural), district, date of onset of the disease, date of notification, date of sample collection, measles vaccination status, date of last vaccination, Patient mode of follow up, $\mathrm{Pa}$ - 


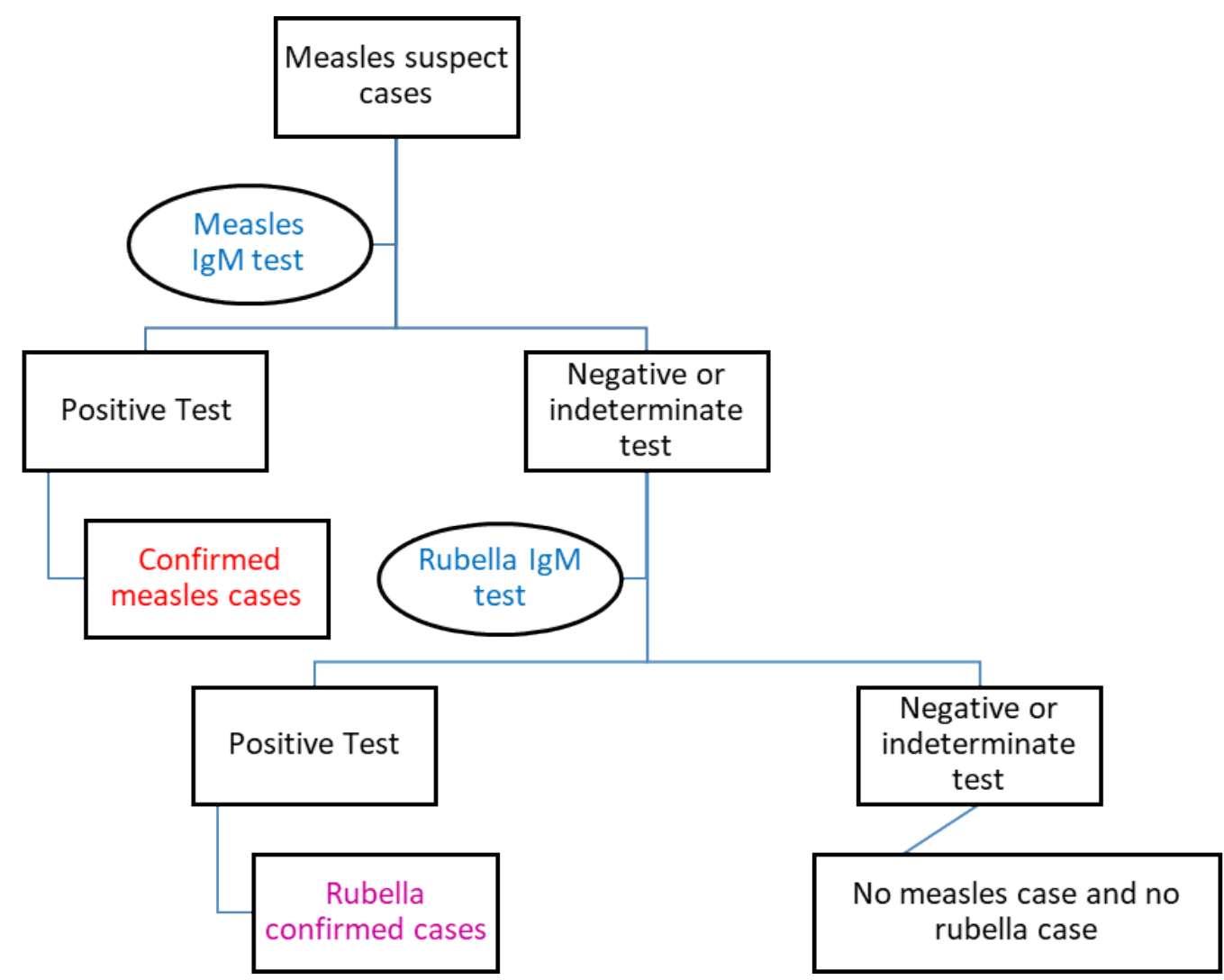

Figure 1: Diagram of procedures for IgM measles and rubella examinations at IPCI lab.

tient Outcome, Sample Condition, Measles IgM Outcome, Rubella IgM Outcome.

\section{Laboratory procedures}

A laboratory samples were taken at the health center level by collecting $5 \mathrm{cc}$ of venous blood. This whole blood was centrifuged or decanted to separate the serum from the red blood cells and collect the serum. This serum was sent to the laboratory at Pasteur Institute of Côte d'Ivoire (IPCI) (for biological confirmation of measles or rubella. This sample is sent to the laboratory in a vaccine carrier between 2 and 8 degrees Celsius.

A blood sample was taken from the first contact with the patient. This specimen must be collected in the 28 days following appearance of the rash.

These samples were tested for IgM antibodies against measles virus in serum. When a suspect case of measles was negative or indeterminate for IgM antibodies against the measles virus, this suspect case was tested for IgM antibodies against rubella virus.

This was an IgM test conducted by biologists at the reference labs of Pasteur Institute in Côte d'Ivoire for the search of recent antibodies against measles and rubella.

Laboratory results were sent to the DCPEV by email (Figure 1).

\section{Data analysis}

All suspect cases of rubella reported in Côte d'Ivoire from January 2012 to December 2016.
A univariate analysis in time (frequency per month), place (frequency of cases by district and region) and person (frequency of sex and age) was conducted to summarize characteristics of suspect cases of rubella and confirmed cases of rubella.

A bivariate analysis were conducted to assess for risk factors differences between rubella cases and negative rubella cases for age group, sex and residence. We reported crude odds ratios (OR) and 95\% confidence intervals.

A multivariate analysis (logistic regression) was performed to build an explanatory model to look for confounding, interaction and linear trends. We reported adjusted odds ratios (OR) and $95 \%$ confidence intervals.

Data from our study were analyzed using Epi Info 3.5.4 and stata version 14 software.

\section{Ethics}

Data was collected during routine surveillance activities for measles. For this routine surveillance data reporting, there is no need for ethic approval in Côte d'Ivoire. However, before collecting patient information, we had the oral consent of the patient or parents or care-givers. Data collection was performed with respect to patient privacy and confidentiality.

\section{Results}

During our study period 4152 suspect cases of measles were notified from the routine surveillance system. Blood sample was collected and tested for measles IgM 
from 3974 suspects cases for which 281 (7.1\%) were measles IgM positive. The remaining samples were either negative for measles IgM antibodies 3656 (92\%) or undetermined 37 (0.9\%). 3693 samples were tested for rubella IgM antibodies. Some $615(16.7 \%)$ of these samples were positive for rubella IgM antibodies, 2962 $(80.2 \%)$ were rubella IgM negative, and $116(3.1 \%)$ were undetermined (Table 1).

Table 1: Repartition of test results.

\begin{tabular}{|l|l|l|}
\hline Diagnostics & Frequency & Proportion \\
\hline Positives Measles IgM & 281 & $7.10 \%$ \\
\hline Positives Rubella IgM & 615 & $15.50 \%$ \\
\hline Negative Rubella IgM & 2962 & $74.50 \%$ \\
\hline Undetermined Rubella & 116 & $2.90 \%$ \\
\hline Total & 3974 & $100.00 \%$ \\
\hline
\end{tabular}

Table 2: Characteristics of suspect cases of rubella $(\mathrm{N}=3693)$.

\begin{tabular}{|l|l|l|l|}
\hline Variables & Categories & Number (n) & Proportion (\%) \\
\hline Age & & 4.7 & {$[1.6 ; 9]^{\star}$} \\
\hline Sex & Male & 1,940 & 52.5 \\
\hline & Female & 1,753 & 47.5 \\
\hline Urban/Rural & Rural & 1,375 & 37.2 \\
\hline & Urban & 2,318 & 62.8 \\
\hline Outcome & Alive & 3,688 & 99.9 \\
\hline & Deceased & 5 & 0.1 \\
\hline
\end{tabular}

("median and Interval interquartile).

Table 3: Univariate analysis of rubella confirmed cases characteristics $(\mathrm{N}=615)$.

\begin{tabular}{|l|l|l|l|}
\hline Variables & Categories & Number (n) & Proportion (\%) \\
\hline Age & & 6.8 & {$[4.2 ; 10]^{*}$} \\
\hline Sex & Male & 331 & 53.8 \\
\hline & Female & 284 & 46.2 \\
\hline Urban/Rural & Rural & 218 & 35.5 \\
\hline & Urban & 397 & 64.5 \\
\hline Outcome & Alive & 612 & 99.5 \\
\hline & Deceased & 3 & 0.5 \\
\hline
\end{tabular}

("Median and Interval interquartile).

\section{Rubella Cases Distribution in General Population}

\section{Characteristics of suspect cases of rubella}

In the suspects' cases, the median age was 4.7 years with interquartile range from 1.6 to 9 years. Among them, 1940 cases (52.5\%) were male and 1753 (47.5\%) females with a gender ratio of 1.1 .

Most of the rubella suspected cases were living in urban areas $(62.8 \%$ ) compared to $37 \%$ in rural areas (Table 2).

\section{Characteristics of confirmed cases of rubella}

For the cases confirmed for rubella IgM antibodies, the median age was 6.8 years and the interquartile range was 4.2 to 10 years.

The majority of rubella positive cases were males (53.8\%) and $46.2 \%$ females with a male to female sex ratio of 1.2 .

Among the rubella positive cases, 397 cases (64.5\%) were living in urban areas while 218 cases (35.5\%) were living in rural areas. Some $99.5 \%$ of the cases survived from rubella infection (99.5\%) while unfortunately 3 cases $(0.5 \%)$ were reported to have died from the disease (Table 3 ).

\section{Distribution of confirmed cases of measles and ru- bella over time}

From 2012 to 2016, we observe two main peaks of positive rubella IgM. The first peak occurs during the month of February 2012 and associated with a peak of positive measles IgM cases.

The second peak occurred in February 2016 but is not associated with any measles peak. The years 2012 and 2016 recorded the highest number of cases with respectively 232 and 192 confirmed rubella cases (Figure 2).

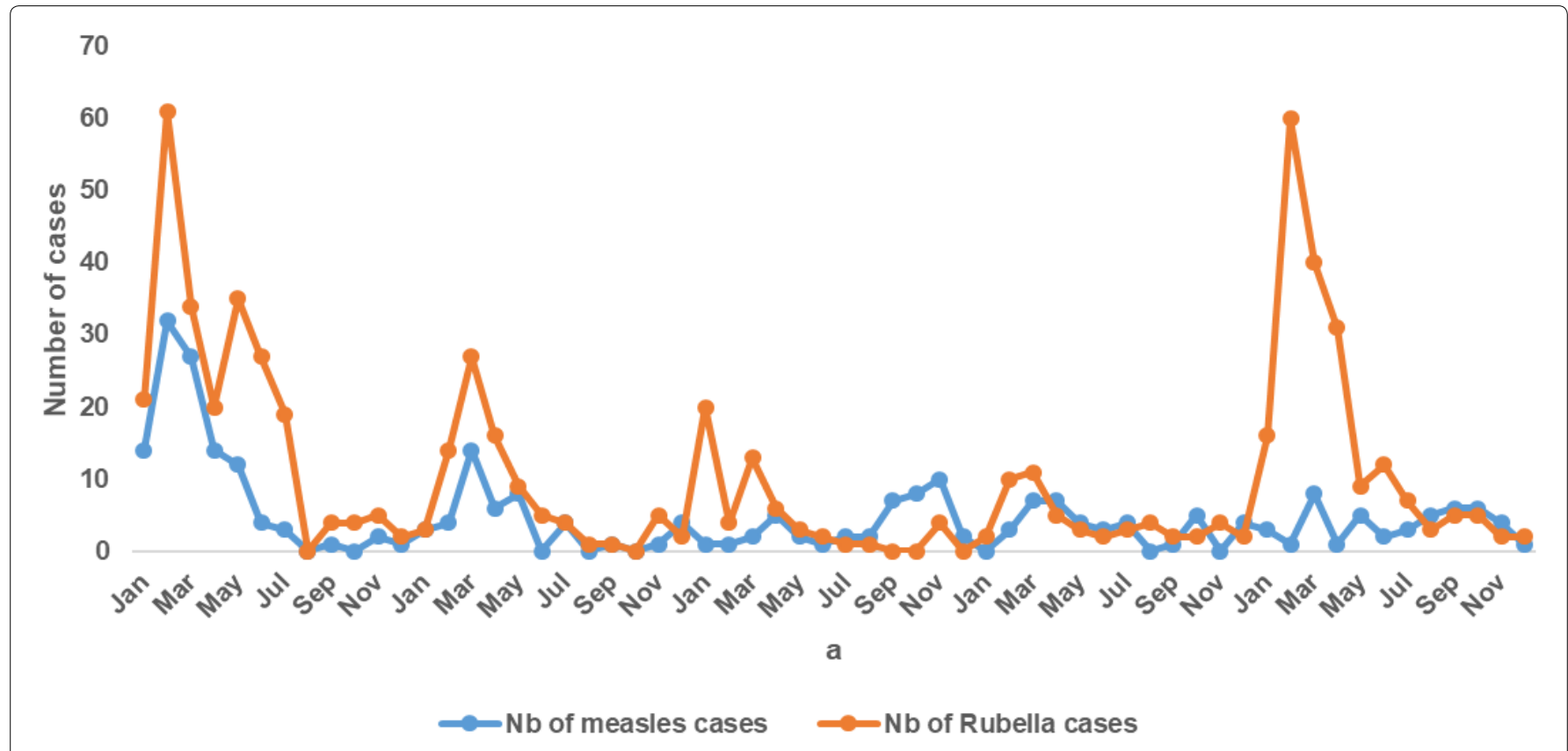

Figure 2: Distribution of rubella confirmed cases and measles confirmed cases from 2012 to 2016 in Côte d'Ivoire. 


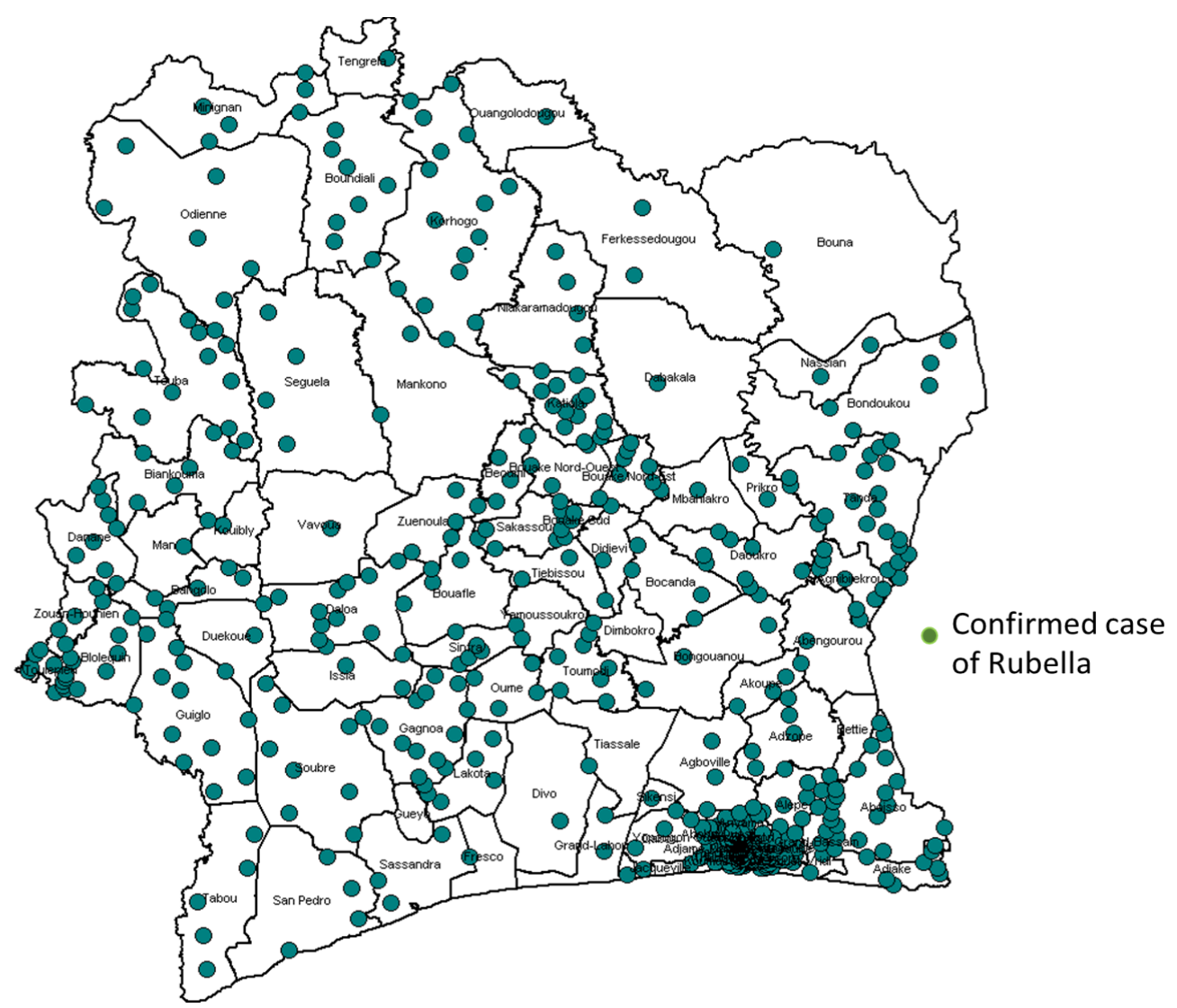

Figure 3: Districts distribution map of rubella confirmed cases in Côte d'Ivoire from 2012 to 2016.

\section{Distribution of cases by districts and regions}

The districts with the highest number of rubella confirmed cases were the districts of Abidjan city (regions of Abidjan 1 Grand Ponts and Abidjan 2). The concerned districts were Abobo Ouest, Abobo Est, Port Bouet Vridi Koumassi, Cocody, Bingerville, Treichville Marcory and Youpogon Est.

These districts were in outbreaks of rubella but these cases have not been investigated.

The other regions that reported large numbers of cases are Cavally-Guemon, Sud Comoe, Bounkani-Gontougo, Poro- Tchologo-Bagoue (Figure 3).

\section{Rubella cases distribution in women of childbearing age}

From the 1753 rubella female suspect cases tested, 24 were positive and aged between 15 years to 49 years, with a proportion of 14 per 1000 . Of the 284 Rubella female confirmed cases, $8.5 \%$ were of reproductive age.

Among these females, the highest numbers of cases were reported in 2012 and 2016 with respectively 7 and 6 cases of rubella.

The regions with the highest number of cases in women of childbearing age were Abidjan 2, Abidjan 1-Grands-Ponts and Goh with respectively 5 cases, 4 cases and 3 cases.

\section{Identify risk factors of rubella}

In the bivariate analysis, children aged from 5 to 14 years were 3.24 times [ $95 \% \mathrm{Cl}(2.68$ - 3.92)] more likely to have rubella compared to children under 5-years-old. People aged from 15 and above were 1.29 times [ $95 \% \mathrm{Cl}(0.9$ -1.84)] more likely to get rubella than children less than 5 -years-old but this relationship was not significant.

There was no difference between male and female regarding rubella infection [OR 1.06, 95\% (0.89 - 1.26)] and no difference between subjects living in rural areas and subjects living in urban areas [OR 1.09, $95 \% \mathrm{Cl}(0.92$ - 1.31)].

In the multivariate analysis, we used logistic regression to look for the model that can best explain rubella infection in Côte d'Ivoire according to the variables available in our surveillance database. Thus, children aged from 5 to 14 years were still at higher risk of rubella compared to children under 5 [OR $3.31 ; 95 \% \mathrm{CI}$ $(2.73-4.00)]$ and the difference between subjects aged 15 and above and children under 5 -years-old were not significant [OR 1.32; 95\% Cl (0.92 - 1.88)].

In this model, people living in urban areas had a higher risk compared to people living in rural areas [OR 1.22; $95 \% \mathrm{Cl}(1.01-1.46)]$ and this difference was significant (Table 4).

\section{Discussions}

Many cases of rubella have been reported in Côte d'Ivoire in recent years where from 2012 to 2016, 615 cases confirmed of rubella were reported through the measles surveillance system but our study has some limitations as we used measles surveillance system data. 
Table 4: Bivariate and Multivariate analysis of rubella suspects cases $(\mathrm{N}=3693)$.

\begin{tabular}{|c|c|c|c|c|c|}
\hline \multirow[b]{2}{*}{ Variables } & \multirow[b]{2}{*}{ Categories } & \multicolumn{2}{|c|}{ Bivariate analysis } & \multicolumn{2}{|c|}{ Multivariate analysis } \\
\hline & & Odds ratio & $95 \% \mathrm{Cl}$ & Odds ratio & $95 \% \mathrm{Cl}$ \\
\hline \multirow[t]{3}{*}{ Age group } & $<5$ years & Ref. & & & \\
\hline & $5-14$ years & 3.24 & $2.68-3.92$ & 3.31 & $2.73-4.00$ \\
\hline & $>=15$ years & 1.29 & $0.9-1.84$ & 1.32 & $0.92-1.88$ \\
\hline \multirow[t]{3}{*}{ Sex } & Female & Ref. & & & \\
\hline & Male & 1.06 & $0.89-1.26$ & & \\
\hline & Rural & Ref. & & & \\
\hline Residence & Urban & 1.09 & $0.92-1.31$ & 1.22 & $1.01-1.46$ \\
\hline
\end{tabular}

In this study, we have 2 major peaks, 2012 and 2016 in 5 years interval. Rubella surveillance data in several developing countries showed that rubella outbreaks occurred every 4-7 years. This is similar to the situation in Europe prior the introduction of the rubella vaccine [6]. Also, the number of cases detected by the measles surveillance system does not necessarily represent all cases of rubella because the case definition of rubella suspected cases is different from that of measles.

In a study in India, $27.7 \%$ (542/1.954) of rubella cases were detected in negative and undetermined cases of measles. Noticing the underreporting of rubella, they recommend the establishment of a rubella surveillance system [7].

According to the $C D C$, rubella surveillance is less sensitive when integrated with measles, as rubella infections is subclinical in $30-50 \%$ of cases [8]. Regarding the surveillance system used to notify rubella cases and information discussed above, we think that this disease is under-reported in Côte d'Ivoire.

In Contrary to rubella, in the same period from 2012 to 2016 , we had a reduction in number of measles cases. In Côte d'Ivoire, the measles vaccine is provided in routine immunization for the first dose and in follow-up vaccination campaigns every 3 to 4 years for the second dose. These vaccinations may have led to a reduction in the number of measles cases.

Children aged 5 to 14 years of age were at higher risk of being infected with rubella compared to children under 5 years of age and those of 15 years and above. Our data differ from those of the CDC in the USA during an outbreak from 1982 to 1992 where the distribution was almost identical in the three age groups [9]. The median age of rubella cases in our study was 6.8 years IR (4.210). During a rubella epidemic in Romania, the median age was 18 years and the most affected age group was 15 to 19 -years-old [10]. We think the fact that these children aged 5 to 14 -years-old were no more protected by maternal antibody against rubella and has the possibility of meeting children at school put them at high of transmission of rubella compare to children less than 5-years-old.

The proportion of male rubella confirmed subjects was higher than that of female cases our data are similar to those in the study in Romania [10].
Our analysis showed that subjects living in urban areas were more likely to get rubella. Indeed, $39 \%$ of rubella cases during the study period were from the capital city area. These results differ from surveillance data in Abia state, Nigeria, where subjects living in rural areas were 1.62 times more likely to have rubella compared to subjects living in urban areas [11].

In our study, $8.5 \%$ of confirmed female rubella subjects were of reproductive age. These women are at risk for CRS. In a sero-epidemiological study of rubella during pregnancy in Côte d'Ivoire in 1993, rubella were present in $82 \%$ of pregnant women and the rubella serology was negative in $18 \%$ of cases. Sero-negative women are at risk of having children with CRS [12].

Rubella epidemics have not been investigated in Côte d'Ivoire. The investigation could help to identify pregnant women among rubella cases and CRS cases. In Japan, the investigation of rubella cases has led to report CRS cases during a rubella outbreak [13].

\section{Conclusion}

The rubella surveillance system in Cote d'Ivoire is not using the WHO recommended Rubella case definition. In such case, our studies could have miss some cases of rubella that do not fit measles cases definition and some measles positive cases could be co-infection cases. However, the national measles case-based surveillance revealed the burden and trends of rubella in Côte d'Ivoire. Although rubella is a childhood disease, a substantial number of women of reproductive age was exposed to primary infection in our study and thus may transmit the virus in utero, with a risk for CRS. Children of 5-14 years-old, adults and people living in urban areas were also at high risk of rubella.

Rubella surveillance could be suboptimal in Côte d'Ivoire as cases are under-reported. CRS is also not included in the current surveillance system.

In the light of these results, the question of introducing rubella vaccine into the national immunization program should be address. These data also suggests that the country should establish a surveillance system for rubella and CRS.

\section{References}

1. Rubella Complications. CDC.

2. Rubéole. OMS WHO. 
3. (2015) Weekly Epidemiological Record. 90: 505-516.

4. Over 700 million children in 49 countries to be protected against measles and rubella. WHO.

5. WHO (2014) Recommended surveillance standard of measles.

6. Cutts FT, Robertson SE, Diaz-Ortega JL, Samuel R (1997) Control of rubella and congenital rubella syndrome (CRS) in developing countries, Part 1: Burden of disease from CRS. Bull World Health Organ 75: 5568.

7. Vaidya SR, Raut CG, Jadhav SM (2016) Laboratory confirmation of rubella infection in suspected measles cases. J Med Virol 88: 1685-1689.

8. Grant GB, Reef SE, Patel M, Knapp JK, Dabbagh A, et al. (2017) Progress in Rubella and Congenital Rubella Syn- drome Control and Elimination - Worldwide, 2000-2016. MMWR Morb Mortal Wkly Rep 66: 1256-1260.

9. Epidemiology of Vaccine Preventable Diseases. Pinkbook Rubella CDC.

10. Umeh CA, Onyi SC (2014) Case based rubella surveillance in Abia State, South East Nigeria, 2007-2011. PeerJ 2: e580.

11. Lazar M, Abernathy $\mathrm{E}$, Chen $\mathrm{MH}$, Icenogle J, Janta $\mathrm{D}$, et al. (2016) Epidemiological and molecular investigation of a rubella outbreak, Romania, 2011 to 2012. Euro Surveill 21.

12. Faye-Kette YH, Sylla-Koko DJ, Akoua-Koffi GC, KacouN'Douba A, Cissel L, et al. (1993) Seroprevalence of rubella in 461 pregnant women in Abidjan (Cote d'Ivoire). Bull Soc Pathol Exot 86: 185-187.

13. Ujiie M, Nabae K, Shobayashi T (2014) Rubella outbreak in Japan. Lancet 383: 1460-1461. 